\title{
MICRO-SHEAR BOND STRENGTH OF NOVEL MDP CALCIUM- FLUORIDE RELEASING SELF-ADHESIVE RESIN CEMENT AFTER THERMOCYCLING
}

\author{
Aliaa Mahrous*, Mohamed M Radwan** and Samah Mohamed Kamel***
}

\begin{abstract}
Purpose: The purpose of the present study was to investigate the micro-shear bond strength and failure mode of novel "MDP calcium-fluoride releasing" self-adhesive resin cement (TheraCem) with tooth structure (enamel\& dentin) and yttrium stabilized zirconia after thermocycling and comparing the results with universal "non-containing MDP" self-adhesive resin cement (Relay X Unicem as a control)
\end{abstract}

Materials and Methods: Enamel and dentin specimens (20 discs each) were obtained by using diamond saw (IsoMet 4000 linear Precision saw, Buehler, USA) with copious water coolant. Twenty zirconia plates were obtained from IPS e.max ZirCad blocks (Ivoclar Vivadent, Schaan, Liechtenstein) and sintered in an inFire HTC speed high-temperature furnace (Sirona Dental System GmbH, Germany). Micro resin cylinders were created on the bonded surface and filled with the tested cements ( $\mathrm{n}=10$ /substrate) group A (control) using non-containing MDP selfadhesive cement Relay X Unicem (3M ESPE) while group B (tested cement) using MDP calciumfluoride releasing self-Adhesive resin cement TheraCem (BISCO, Schaumburg, Illinois, USA). Cements were left to self-cure for 5 minutes. All the specimens were thermocycled for 5,000 cycles (Thermocycler 1100 SD Mechatronik, GmbH). Micro-shear bond strength was measured using universal testing machine and debonded surfaces were examined for failure mode analysis with all morphological and ultrastructure changes using Scanning Electron Microscope (Quanta 250 Field Emission Gun, Netherlands) attached with EDX Unit (Energy Dispersive X-ray Analyses). The results were statistically analysed.

Results: TheraCem (tested cement) had a slightly higher (mean \pm SD) $\mu$-SBS (Mpa) value than RelyX (control) (18.96 $\pm 4.36,12.18 \pm 3.13$; respectively) ( $\mathrm{P}=0.177)$. Within enamel, TheraCem $(6.46 \pm 1.37)$ had a significantly higher $(\operatorname{mean} \pm \mathrm{SD}) \mu$-SBS $(\mathrm{Mpa})$ value than RelyX $(3.04 \pm 0.99)$ $(\mathrm{P}=0.002)$. Similarly, TheraCem in dentine $(10.67 \pm 1.27)$ had a significantly higher (mean $\pm \mathrm{SD})$ than RelyX (6.46 \pm 1.74$)(\mathrm{P}=0.014)$. As for zirconia, TheraCem $(39.76 \pm 1.18)$ had a significantly higher $($ mean \pm SD) $\mu$-SBS $(\mathrm{Mpa})$ than RelyX $(27.04 \pm 1.92)(\mathrm{P}<0.001)$

\footnotetext{
* Lecturer Fixed Prosthodontics Department, Faculty of Dentistry, Fayoum University, Egypt.

** Lecturer Fixed Prosthodontics Department, Faculty of Dentistry, Beni Suaf University, Egypt.

*** Lecturer Oral Biology, Faculty of Dentistry, Modern Science and Arts University (MSA), Egypt.
} 
Conclusion: using MDP calcium-fluoride releasing self-adhesive resin cement (TheraCem) may improve bond strength to all tested substrates (enamel, dentin and zirconia).

Clinical significant: TheraCem self-adhesive resin cement can be considered as promising cement for many clinicians. Further clinical studies are required to provide long term clinical success data.

Keywords TheraCem self-adhesive, MDP Calcium-fluoride Releasing adhesives, Relay X Unicem, enamel, dentin, zirconia, thermocycling, micro-shear bond strength, failure mode.

\section{INTRODUCTION}

The clinical success of any adhesive resin cement can be primarily evaluated by proper marginal sealing of the adhesive with both the restoration and tooth structure. Ideal adhesive cement must have sufficient strength, biocompatibility, good wettability and dissolution resistance. In conventional resin cements, a pretreatment procedure is required to achieve adhesion but the procedure is complicated as bond strength is weakened when the moisture-proofing or dentin wettability is not properly maintained ${ }^{(1,2)}$. A selfetching system was developed to use a new type of adhesive resin cements that eliminated the need of separate priming step ${ }^{(3)}$, simplified the treatment procedures and prevented the collapse of the collagen fibers in the dentin through acid etching. It has recently attracted interest of researchers and clinicians as it was recommended not only for tooth tissues bonding and composites but also for most of the indirect restorations such as zirconia and glassceramics $^{(3,4)}$.

However, the permeation of moisture through the adhesives can cause the bond strength to deteriorate when hardening is delayed. To overcome this problem, self-adhesive resin cements (SARC) that combine the adhesives and cements were developed $^{(5)}$. These cements became the most widely used adhesives, as they offer the mechanical, aesthetic and the adhesive advantages of typical resin cements and also do not require pretreatments, due to their acidic functional monomer ${ }^{(6)}$.
The basic bonding mechanism to tooth structure depends on mineral exchange by resin monomers that form micro-mechanical interlocking in the surfaces $^{(2)}$. Bonding durability of these adhesives have been studied to explain the adhesion mechanism with tooth structure (enamel \& dentin), it was reported that the type of acidic monomer in adhesives may play a major role in producing a stable chemical bond,with MDP containing materials making the most stable bond. MDP bonds to hydroxyapatite and results in an intermediate layer consisting of two MDP molecules, with methacrylate groups being directed towards each other, while phosphate groups are directed away from each other and Calcium salts are deposited between the layers of their phosphate groups ${ }^{(5,6)}$.

On the other side, adhesive resins with added calcium and phosphate ions can act as a reservoir on tooth surface as these ions are released in acidic media to facilitate the remineralization that may positively affect the shear bond strength with the tooth surface ${ }^{(7,8)}$.

With the continuous evolvement of ceramic materials, they have become the first option for many clinical cases, especially with increasing the patient's esthetic demands. On the other side, the use of ceramics in long span restorations have been limited till the introduction of high strength ceramics which possess unique and excellent mechanical properties. Nowadays, zirconia is commonly selected in restorations all over the world. It's well documented that, zirconia restorations can be cemented by any traditional 
cements ,but the reliable adhesion of adhesive resin cement to zirconia improves the marginal seal, retention and subsequently the fracture resistance of the restoration ${ }^{(9)}$.

During the last 15 years, many studies discussed the bonding challenges of zirconia restorations that face clinicians daily; zirconia has an inert surface which complicates obtaining the required microretention bond ${ }^{(10,11)}$.

Many trials to increase zirconia adhesive bond strength were reported. Silica coating, air borne particle abrasion, laser and acid etching have all been tested with no significant improvement in resinzirconia bond strength $^{(9,12)}$. Researches have reported that the functional monomer 10-methacryloyloxydecyl-dihydrogen-phosphate (10-MDP) containing primers that may positively affect resin-zirconia bond strength.

The improvement of the bond strength between zirconia and resin substrate is obtained through chemical bonding with the oxide layer ${ }^{(13,14)}$. MDP monomers contain functional phosphate ester group that directly form a chemical bond to metal oxides of zirconia. The first MDP containing material was owned by Kurary Company ${ }^{(15)}$.

Interestingly, many studies discussed how MDP containing primer, conditioner or adhesive cement may affect the zirconia bond strength ${ }^{(11,16)}$.

Bond strength of MDP containing materials may be affected after artificial aging or thermocycling as MDP molecules lack bonding stability ${ }^{(9,11)}$.

The aim of the present study was to investigate the micro-shear bond strength of new MDP calciumfluoride releasing self-adhesive resin cement (TheraCem) with tooth structure (enamel, dentin) and zirconia after thermocycling in comparison to non-containing MDP self-adhesive resin cement (RelayX Unicem). Furthermore, analyze the debonded surfaces to detect the failure mode with all structural and morphological changes.
The null hypothesis tested was that, firstly; the micro-shear bond strength of new MDP calciumfluoride releasing self-adhesive resin cement (TheraCem) will be significantly higher than noncontaining MDP self-adhesive resin cement (Relay $X$ Unicem). Secondly, MDP calcium-fluoride releasing self-adhesive resin cement will have higher bonding to enamel, dentin and zirconia substrates.

\section{MATERIALS AND METHODS}

The study was divided into 2 different parts; the first part was concerned with enamel and dentin bonding evaluation with different types of cement while the other part was related to zirconia ceramic bonding evaluation. All groups $(\mathrm{n}=10)$ were subjected to micro-shear bond strength test and failure mode analysis with all morphological and ultrastructure changes on debonded surface.

\section{Enamel and Dentin specimen preparation}

A total of forty freshly extracted non carious lower permanent molars were collected for the present study. The selected molars should be with intact enamel surface, no cracks or previous treatment with chemical agents. All molars were ultrasonically cleaned to remove all debris, calculus and periodontal tissues. The roots were trimmed off using diamond saw (IsoMet 4000 linear Precision saw, Buehler, USA) with copious water coolant. The teeth were stored in $0.5 \%$ chloramine solution in refrigerator at $4^{\circ} \mathrm{C}$ and used within 1 month following extraction.

Regarding the enamel specimen preparation; twenty molars were used for obtaining the enamel discs by sectioning the teeth into bucco-lingual using diamond saw (IsoMet 4000 linear Precision saw, Buehler, USA) with copious water coolant. As for the dentin specimen preparation; the occlusal surfaces of the other twenty molars were cut to the mid coronal part of dentin without exposing pulpal tissue. 
All prepared enamel and dentin specimens were embedded in split cylindrical tefflon molds filled with self-cure acrylic resin with the bonding site facing upward for testing. After complete setting of the self-cure resin, molds were carefully removed and specimens were kept in $100 \%$ humidity at $4^{\circ} \mathrm{C}$ till the cementing time.

Prior to cementing procedure, the enamel and dentin specimens were polished with wet silicon carbide ( $\mathrm{SiC}$ ) sandpaper size 600 grit (Sailbrand, China) for 30 seconds to standardize a smear layer on the bonded surface. The silicon carbide paper was changed after each 10 specimens. Finally, all specimens were washed with distilled water and dried with oil-free air.

\section{Zirconia specimen preparation}

A total of twenty zirconia ceramic plates were obtained from IPS e.max ZirCad blocks (Ivoclar Vivadent, Schaan, Liechtenstein) using diamond saw (IsoMet 4000 linear Precision saw, Buehler, USA) with copious water coolant. Zirconia specimens were sintered in an inFire HTC speed high-temperature furnace (Sirona Dental System $\mathrm{GmbH}$, Germany). The plates were embedded in split cylindrical tefflon molds filled with self-cure acrylic resin with the bonding site facing upward for testing. The bonded zirconia surfaces were all polished with 600, 800 and 1200 grit silicon carbide paper (Sailbrand, China) under continuous use of water coolant. Finally, zirconia specimens were ultrasonically cleaned in distilled water for 5 minutes before bonding procedures.

\section{Bonding procedures for all substrates}

A $0.75 \mathrm{~mm}$ diameter and $1 \mathrm{~mm}$ height resin cement cylinders were created using translucent mold (Tygon tubing, TYG-030; SaintGobain Performance Plastic, Clearwater, FL) positioned over specimens and filled with the tested cements; group A using dual cure self-adhesive cement
Relay X Unicem (3M ESPE) while group B using dual cure self-Adhesive resin cement TheraCem (BISCO, Schaumburg, Illinois, USA). All tested groups were left for self-cure for 5 minutes according to manufactures instructions. Resin cement cylinders were exposed by removing the molds using surgical scalpel blade (No. 15; SwannMorton, Sheffield, UK). Before testing, all resin cement micro-cylinders were checked for bonding interface defects.

All the specimens ( $\mathrm{n}=10$ for each used cement) (Enamel, Dentin, Zirconia), were immersed in water at $37{ }^{\circ} \mathrm{C}$ for 24 hours before being thermocycled using a thermocycling device (Thermocycler 1100 SD Mechatronik, $\mathrm{GmbH}$ ) for 5,000 cycles between $5{ }^{\circ} \mathrm{C}$ and $55^{\circ} \mathrm{C}$ with 60 seconds dwell time at each bath before testing.

\section{Micro-shear bond strength test}

Each Specimen with its bonded resin microcylinder was fixed with tightening screws to lower fixed compartment of mechanical testing machine (Model 3345; Instron Industrial Products, Norwood, MA, USA) with a $5 \mathrm{KN}$ loadcell. A mono-bevel chisel ended rod was aligned with the loading axis of the upper movable compartment of the machine at a crosshead speed of $0.5 \mathrm{~mm} / \mathrm{min}$ to apply shearing load as close as possible to the resin micro-cylinder base. The load required for debonding was recorded in Newton using computer software (Instron ${ }^{\circledR}$ Bluehill Lite Software). MicroShear bond strength ( $\mu$-SBS) was calculated from the following equation $[\tau=\mathrm{P} / \pi \mathrm{r} 2]$ Where; $\tau=$ $\mu$-shear bond strength $(\mathrm{MPa}), \mathrm{P}=\operatorname{load}$ at failure $(\mathrm{N})$, $\pi=3.14$ and $r=$ radius of micro-cylinder $(\mathrm{mm})$.

\section{Scanning Electron Microscope}

After debonding, three specimens of each group were randomly selected and the debonded surfaces were examined for failure mode analysis with all morphological and ultra-structural changes using 
Scanning Electron Microscope (Quanta 250 Field Emission Gun, Netherlands) attached with EDX Unit (Energy Dispersive X-ray Analyses), with accelerating voltage of $30 \mathrm{~K} . \mathrm{V}$., magnification $14 \mathrm{x}$ up to 1000000 and resolution for Gun.1n with a secondary electron detector. Failure mode was categorized as adhesive, cohesive or mixed.

\section{RESULTS}

Numerical data were explored for normality by checking the data distribution, calculating the mean and median values and using Kolmogorov-Smirnov and Shapiro-Wilk tests. Data showed parametric distribution, so it was represented by mean and standard deviation (SD) values. Independent t-test was used for different intergroup comparisons. The significance level was set at $\mathrm{P} \leq 0.05$ for all tests. Statistical analysis was performed with IBM ${ }^{\circledR}$ SPSS ${ }^{\circledR}$ (SPSS Inc., IBM Corporation, NY, USA) Statistics Version 25 for Windows.

The results showed that micro-shear bond strength (table 1) of the tested cement TheraCem $(18.96 \pm 4.36)$ had a slightly higher $($ mean \pm SD) value than RelyX Unicem (control) $(12.18 \pm 3.13)$ $(\mathrm{P}=0.177)$

TABLE (1) Mean and standard deviation (SD) for $\mu$-SBS (Mpa) for different types of cements

\begin{tabular}{|c|c|c|}
\hline \multicolumn{2}{|c|}{ Type of cement $($ mean \pm SD) } & \multirow{2}{*}{ P-value } \\
\cline { 1 - 2 } RelyX (control) & TheraCem & \\
\hline $12.18 \pm 3.13$ & $18.96 \pm 4.36$ & $0.177 \mathrm{~ns}$ \\
\hline
\end{tabular}

*; significant ( $p \leq 0.05) \quad n s ;$ non-significant ( $p>0.05)$

For all tested cements, dentin showed a significantly higher $\mu$-SBS (Mpa) than enamel (table 2). RelyX Unicem (control) dentine showed a significantly higher $\mu$-SBS $($ mean $\pm \mathrm{SD})$ value than enamel $(6.46 \pm 1.74,3.04 \pm 0.99$; respectively)
$(\mathrm{P}=0.031)$. The same applies to TheraCem, (10.67 $\pm 1.27,6.46 \pm 1.37$; respectively) $(\mathrm{P}=0.001)$.

Within enamel, TheraCem $(6.46 \pm 1.37)$ had a significantly higher $\mu$-SBS $($ mean $\pm \mathrm{SD})$ value than RelyX (3.04 \pm 0.99$)(P=0.002)$. Similarly, TheraCem within dentine $(10.67 \pm 1.27)$ had a significantly higher $\mu$-SBS $($ mean \pm SD) than RelyX $(6.46 \pm 1.74)$ ( $\mathrm{P}=0.014$ ), (figure 1). Regarding zirconia, $\mu$-SBS (Mpa) in TheraCem had a significantly higher $($ mean \pm SD) than RelyX (39.76 $\pm 1.18,27.04 \pm 1.92$; respectively) $(\mathrm{P}<0.001)$, Figure (2).

TABLE (2) Mean \pm standard deviation (SD) of $\mu$-SBS (Mpa) for enamel and dentine within each type of cement

\begin{tabular}{|c|c|c|c|}
\hline \multirow{2}{*}{$\begin{array}{c}\text { Type of } \\
\text { cement }\end{array}$} & \multicolumn{2}{|c|}{$\begin{array}{r}\text { Type of bonding substrate } \\
(\text { mean } \pm \text { SD) }\end{array}$} & \multirow{2}{*}{ P-value } \\
\cline { 2 - 3 } & Enamel & Dentine & \\
\hline RelyX & $3.04 \pm 0.99^{\mathrm{A}}$ & $6.46 \pm 1.74^{\mathrm{A}}$ & $0.031^{*}$ \\
\hline TheraCem & $6.46 \pm 1.37^{\mathrm{B}}$ & $10.67 \pm 1.27^{\mathrm{B}}$ & $0.001^{*}$ \\
\hline
\end{tabular}

*; significant $(p \leq 0.05) \quad n s ;$ non-significant $(p>0.05)$.

Different superscript letters within the same column indicate a statistically significant difference.

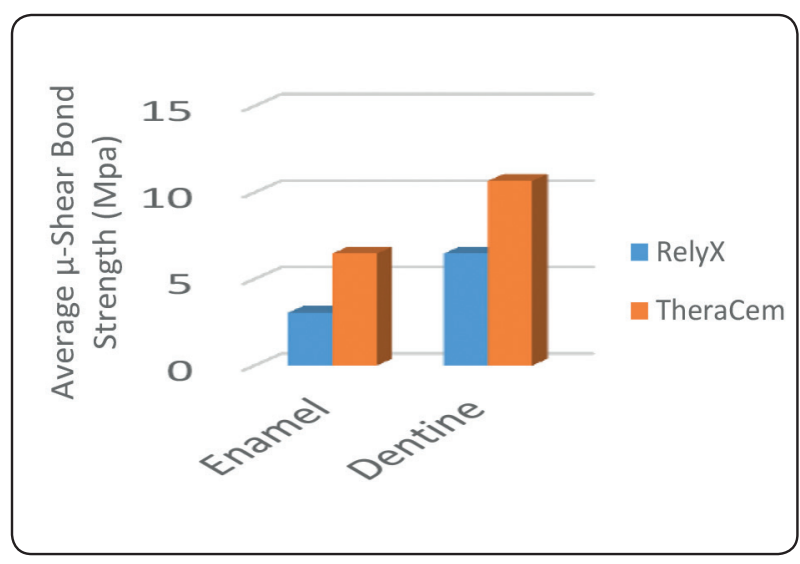

Fig. (1): Bar chart showing average $\mu$-SBS (Mpa) for different types of cement with enamel and dentine. 


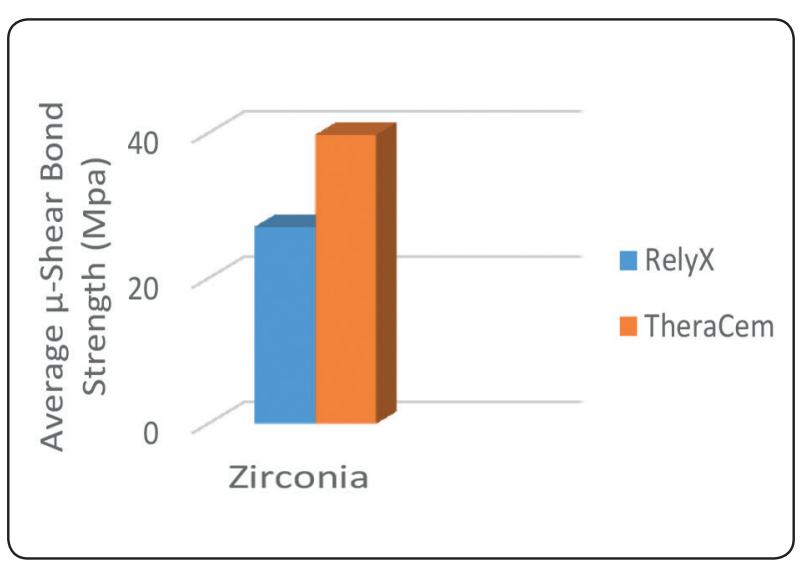

Fig. (2): Bar chart showing average $\mu$-SBS (Mpa) for different types of cement with zirconia
Scanning Electron Microscope with energy dispersive X-ray spectroscopy (SEM/EDX) of the specimens (figure 3 ) showed that in all tested enamel groups, failure mode of enamel debonded surface was mixed with many detached cement areas. Less homogenous cement spread in RelyX group than in TheraCem group. EDX analysis revealed increase in calcium and phosphorous concentrations in TheraCem group indicating formation of calcium phosphate depositions at the surface.

Regarding the debonded dentin surface (figure 4), the failure mode was mixed in RelyX group with some empty dentinal tubules while TheraCem group showed complete obliteration of the dentinal tubules

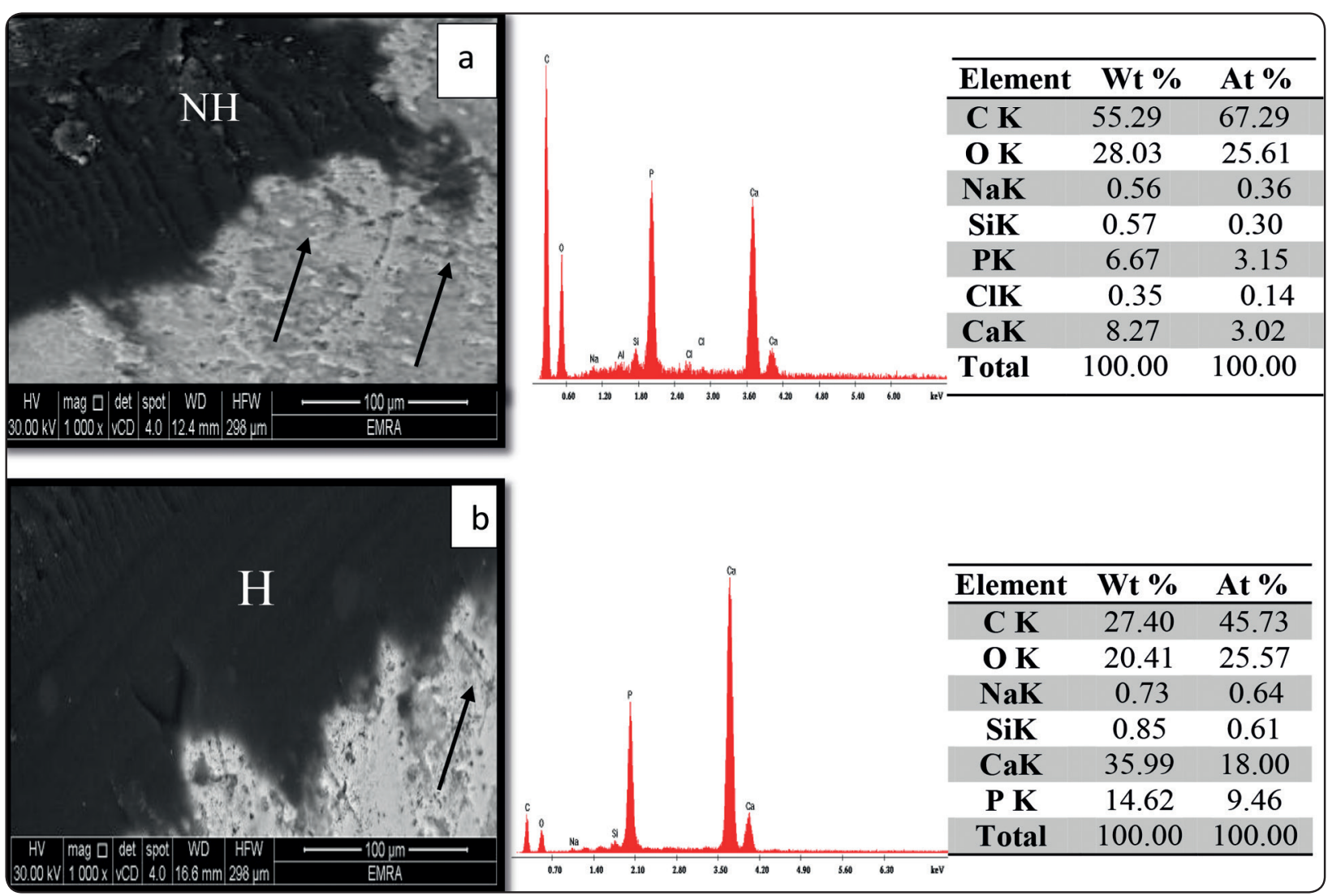

Fig. (3): Representative SEM (x1000 magnification) morphologic characterization of debonded Enamel surface with cement precipitations formed over the enamel rods using RelayX (figure 3a) and TheraCem (figure 3b). TheraCem (figure 3b) was spreading homogenously over the enamel surface $(\mathrm{H})$, while this homogeneity was less $(\mathrm{NH})$ in RelyX (figure 3a) some parts of the cement in both groups were scattered over the enamel surface with detached areas (black arrows) denoting that the mode of failure in both of them is mixed. EDX spectrum was obtained from the precipitates in the field of view. Semiquantitative chemical composition presented in the table shows their $\mathrm{Ca} / \mathrm{P}$ ratio. 
with resin tags indicating for cohesive failure. EDX analysis showed increase in calcium concentrations in TheraCem group explaining formation of calcium precipitations at the surface. The failure mode was cohesive in most specimens within the resin cement in TheraCem but mixed in RelyX.

Zirconia surface (figure5a) showed small area of RelyX cement covering less than $50 \%$ of the surface with few islands on the surface. TheraCem (figure 5b) was spreading more homogenously over the surface and covered more than $50 \%$ of the surface with more islands of cement distributed over the remaining area. The islands in TheraCem were many and smaller but distributed in more areas as it was dragged off the surface explaining the cohesive failure in most examined samples. Higher magnifications showed difference in grain size of RelayX group (fig5c) and this difference decreased with the TheraCem group (figure 5d) which aided in better flowbility and adhesion to zirconia. Failure mode was mainly mixed in both cements with increased cohesive ratio at TheraCem.

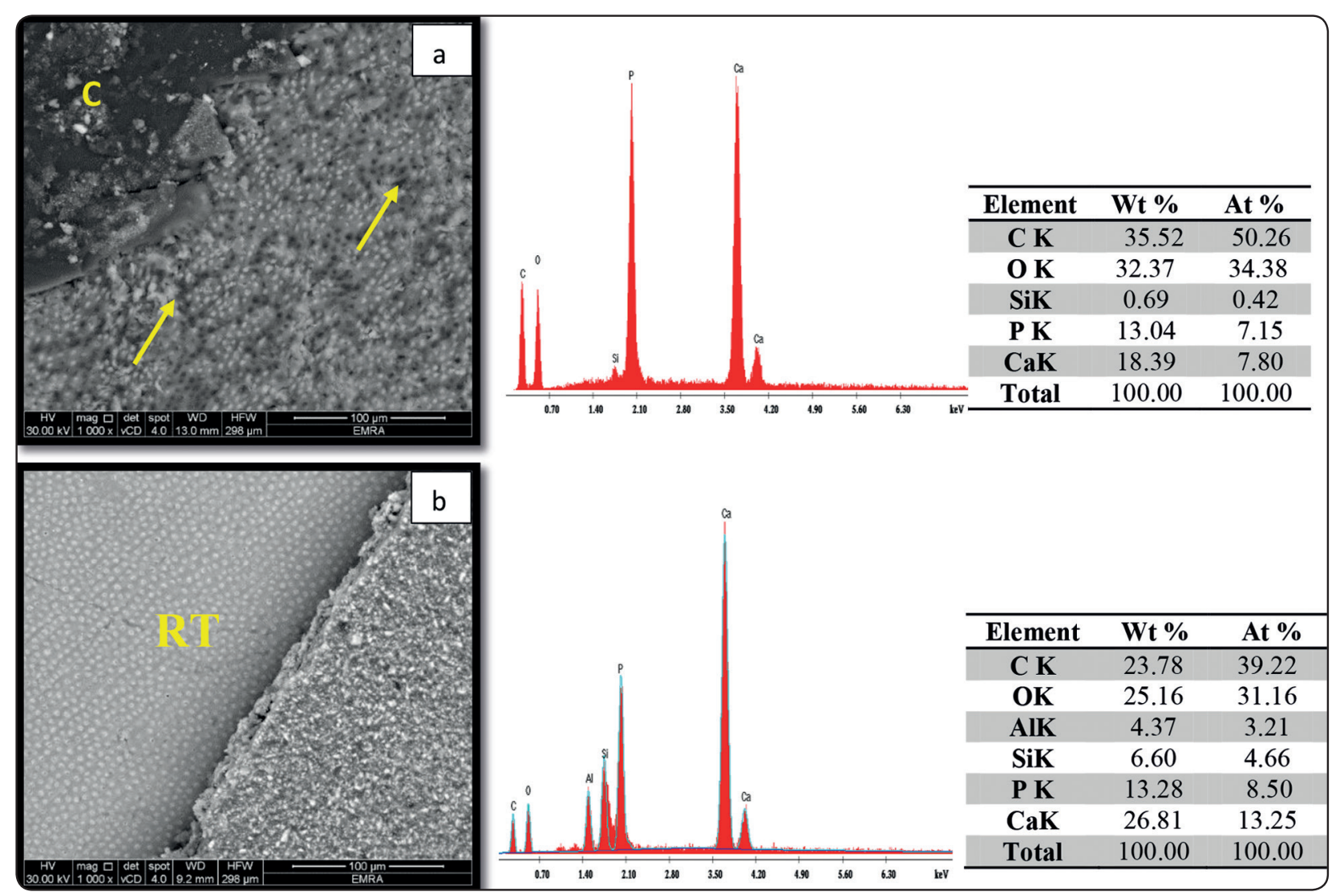

Fig. (4): Representative SEM (x1000 magnification) morphologic characterization of the debonded Dentin surface with RelayX (figure 4a) and TheraCem (figure 4b). The dentinal tubules in RelyX (figure 4a) were not completely obliterated by resin tags (yellow arrows) with mixed failure mode. TheraCem (figure $4 \mathrm{~b}$ ) showed complete obliteration of dentinal tubules by the resin tags (RT) with cohesive failure mode. EDX spectrum was obtained from the precipitates in the field of view. Semiquantitative chemical composition presented in the table shows their $\mathrm{Ca} / \mathrm{P}$ ratio. 


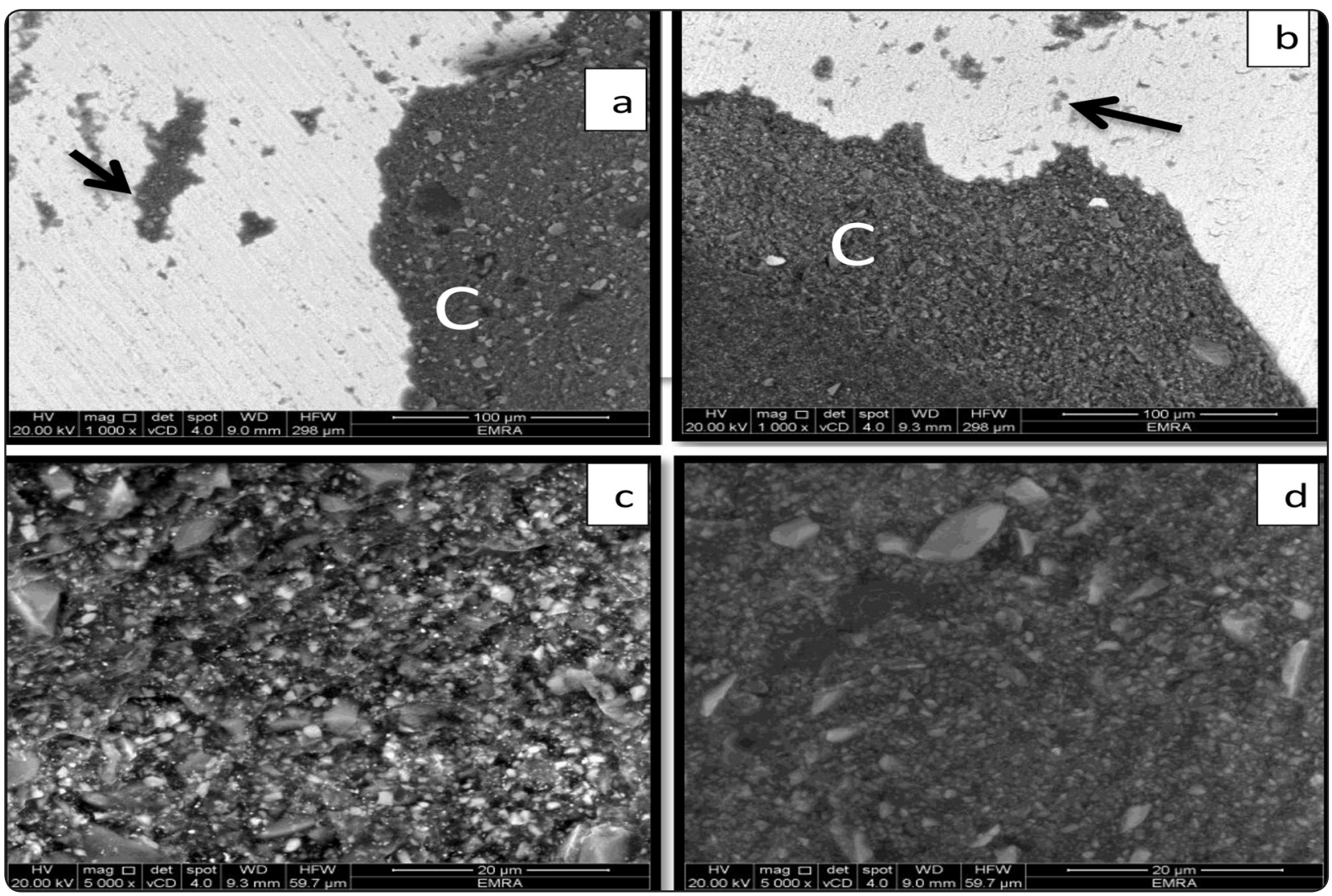

Fig. (5): Representative SEM (x1000 magnification) morphologic characterization of debonded zirconia surface with cement precipitations on surface of RelayX (figure5a) and TheraCem (figure5b). zirconia surface (figure5a) showed small area of RelyX cement (C) covering less than $50 \%$ of the surface with few islands (black arrow) on the surface. TheraCem (figure $5 \mathrm{~b}$ ) was spreading more homogenously over the surface and covered more than $50 \%$ of the surface with more islands of cement distributed over the remaining area (black arrow). The islands in Theracem were many and smaller but distributed in more areas as it was dragged off the surface explaining the cohesive failure in most examined samples. Higher magnifications (x 5000) showed difference in grain size of RelayX group (fig5c) and this difference decreased with the TheraCem group (figure5d) which aided in better flowbility and adhesion to zirconia.

\section{DISCUSSION}

Due to the outstanding efficacy of resin cements, their clinical application has increased and their simplicity was reported in many vivo and in vitro researches.

Nevertheless, it is important to know that the market has many varieties of commercial brands of resin cements. To clarify, each category of adhesive resin cement has its individual unique chemical composition that determines its interactions with the tooth structure and different restorative materials ${ }^{(9)}$.
Self-adhesive resin cements can provide a reliable bond to dental structures while eliminating the etching procedure and need for bonding application. As their application is accomplished in one step, thus saving time, they became very attractive to many clinicians. The bonding mechanism of these adhesive resins relies on chemical reaction and bonding rather than on micromechanical retention ${ }^{(17)}$.

After the application of self-adhesive cement, the phosphoric acid methacrylate can cause demineralization of the hard dental tissues ${ }^{(2)}$. 
However, despite the initial acidic $\mathrm{pH}(\mathrm{pH}<2.0)$, the tooth tissues can only demineralized superficially.

As a result of the reaction between the phosphate groups and alkaline fillers with the hydroxyapatite from enamel and dentin, an increase in $\mathrm{pH}$.(up to 7.0) that neutralizes the acidity of the adhesive resin can be observed

The resin acid groups chelate the calcium ions of the hydroxyapatite, creating true chemical bonding. In addition, carboxylic groups of poly-alkenoic acid (found in RelyX Unicem) form chemical ionic bonds with calcium present in the hydroxyapatite, positively influencing the chemical bonding ${ }^{(3,18)}$.

Self-adhesive resin cements can partially dissolve the smear layer while keeping the smear plugs within the opened dentinal tubules ${ }^{(3,6)}$. A thick smear layer may negatively affect the bond strength of self-adhesive cements, since the chemical bond is achieved with hydroxyapatite. Dentin acid etched with phosphoric acid before the application of self-adhesive resin cement is detrimental to bond strength and must be avoided ${ }^{(19)}$.

Conversely, the application of mild acidic agents, such as $25 \%$ poly-acrylic acid (same dentin conditioner used for glass-ionomer cements), might remove the superficially loose bound fraction of the smear layer, thus improving adhesion ${ }^{(20)}$.

The current study tests the micro-shear bond strength using bonded cross-sectional areas of $1 \mathrm{~mm}$.

It can be considered as a simple method that permits efficient analysis of adhesive resin, regional and depth profiling of a variety of substrates, and conservation of teeth. When compared with microtensile bond strength, it was reported that the specimen is pre-stressed only by mold removal ${ }^{(21)}$.

This study examines the micro-shear bond strength of novel self-adhesive cements (tested cement: TheraCem) and compares it with the universal popular self-adhesive resin cement (control: RelyX Unicem). The micro-shear bond strength with different substrates (enamel, dentin and zirconia) resulted in no statistically significant differences among the groups (table 1). Thus, the $1^{\text {ST }}$ null hypothesis of the present study was rejected. The relative low bond strength values obtained in both groups ${ }^{(22-224)}$ can be explained by the fact that "self-adhesive cements do not dissolve the smear layer and interact only superficially with the tooth structure". (25)

A few factors may contribute to this possibility. $1^{\text {st }}$ the light-cured cement shows a high viscosity with limited penetration/interaction time ${ }^{(26)} \cdot 2^{\text {nd }}$ selfadhesive cements need to be applied with some pressure $^{(27)}$; as to have a proper intimate adaptation to the surfaces ${ }^{(28)} \cdot 3^{\text {rd }}$ the design of the micro-shear testing requires resin cylinders to be bonded to the tooth ,but light curing of these relatively thick specimens might result in a high polymerization stress, causing resin contraction away from the bonded surface ${ }^{(21)}$.

Moreover, micro-shear bond strength of selfadhesive resin cement is attributed to the type of multifunctional monomers ${ }^{(9,11,15)}$, which might differ according to cement type. RelyX Unicem contains methacrylate phosphoric esters, whereas TheraCem contains a hydrophilic monomer (10MDP) and calcium fluoride, both evidently having a low initial $\mathrm{pH}$. Normally, the cements should be capable of demineralizing and infiltrating the tooth structure.

Nevertheless, only varying areas of etching have been observed and almost no distinct demineralization or hybrid layer formation have been found after using self-adhesives. Therefore, chemical rather than micromechanical bonding is responsible for the dental adhesion of the resin components, especially 10 MDP, which was shown to chelate the calcium ions of hydroxyapatite crystals , and this can explain the slight increase in micro-shear bond strength of TheraCem cement (MDP containing) than RelayX (non MDP containing) ${ }^{(29,30)}$. 
On the other hand, Han et al. ${ }^{(29)}$ demonstrated that self-adhesive cements do not achieve a neutral $\mathrm{pH}$, and that maintaining a low initial $\mathrm{pH}$ for an extended period can adversely affect the dentin bonding of these cements. Nakamura et al. ${ }^{(31)}$ found that certain self-adhesive cements have excellent mechanical properties in terms of flexural strength, elastic modulus, and water absorption, which attributed to the presence of 10 MDP.

MDP is the acidic monomer in Panavia (Kurary Dental, Japan), and its presence has enhanced the bonding to the tooth structure as reported in many researches. And this is reported in the current study (table1, 2).

The presence of adhesive monomer MDP in the mass of resin cement (TheraCem) yields a stronger micro-shear bond than the other non MDP containing resin cement (RelyX Unicem).

The 1st part of the study investigates the microshear bond strength of self-adhesive resin cement (TheraCem) and (RelyX) with tooth structure (enamel, dentin). It is found that the bond strength values observed for the enamel were low for both groups (table 2). The reason is that the bond strength of self-adhesive resin cements is lower than that of etch-and-rinse resin cements or 10-MDP self-etch resin cements. This was supported by using SEM to detect failure modes; failure modes in enamel specimens were mixed but the adhesive mode was prominent. Subsequently, pretreatment of enamel with strong acid, such as $35 \%$ phosphoric acid, is highly recommended to improve the bonding.

The results found that the micro-shear bond strength values of TheraCem group were significant higher than Rely $\mathrm{X}$ in both enamel and dentin substrates (table 2, figure 1). Self-adhesive cements initially have a low $\mathrm{pH}$ and the acid groups connect with calcium hydroxyapatite to form a stable bond between the methacrylate network and the tooth. Sodium, calcium, fluoride, and silicate ions that are released by alkaline particles neutralize the remaining acid groups and the presence of calcium hydroxide seems to accelerate the neutralization ${ }^{(32,33)}$. TheraCem cements tested in this study have calcium fluoride and MDP in their composition.

In the present study, TheraCem shows a higher bonding performance regardless of the bonding substrate. The interaction between resin cement and dentin creates a micromechanical interlocking that is considered as the fundamental principle of adhesion to the tooth substrate, based on an exchange process where inorganic tooth material is exchanged for synthetic resin $^{(31)}$.

This process involves 2 phases. One phase consists of the removal of calcium phosphates, by which micro-irregularities are exposed at the dentin surface. The subsequent so-called hybridization phase involves infiltration and in situ polymerization of resin within the produced micro-irregularities micromechanical interlocking is believed to be a prerequisite in achieving good bonding within clinical circumstances, the potential benefit of additional chemical interactions between functional monomers and tooth-substrate components has regained attention recently.

According to the Adhesion-Decalcification concept, specific functional monomers (calcium and florid) within TheraCem resin cement can ionically interact with Hydroxyapatite ${ }^{(34)}$. Such molecules are able to etch/infiltrate the dentin and react with Hydroxyapatite, generating calcium ions with a reduced binding energy. These ions act as an electron acceptor, enabling chemical interaction with the composite. This way, the micromechanical interlocking and chemical binding with Hydroxyapatite are thought to synergistically provide the ultimate adhesion of the material.

In addition to the functional monomers, there are other components in TheraCem that are a key to their bonding performance. For example, rheological modifiers were incorporated in TheraCem to increase the flow ability of the cement, which is 
thought to improve the wettability of the cement to the substrate. ${ }^{(30)}$. In fact, the mean bond strength of TheraCem to dentin is significantly higher than (RelyX) .This result is supposed to be directly related to the amount of calcium ions $(\mathrm{Ca} 2+)$ available for bonding, which varies significantly according to tooth region (enamel or dentin ) (27). However, the difference in bond strength was explained again by failure mode and morphological changes of debonded surfaces demonstrated by the SEM (figure 3, 4).

The $2^{\text {nd }}$ part of the study deals with micro-shear bond strength of zirconia to different cements. It is concluded that zirconia shows significantly higher micro-shear bond strength (figure 2) with TheraCem group (MDP containing) than with RelyX group (non MDP containing), this was also supported by failure mode analysis using SEM (figure 5), where TheraCem showed more cohesive failure than RelyX. The null $2^{\text {nd }}$ hypothesis was accepted. These were in agreement with results in the literature, demonstrating that 10-MDP can improve the bonding effectiveness of conventional resin cement to zirconia restorations ${ }^{(13-16)}$. Also, many studies reported no significant difference in SBS before and after thermo-cycling for 10-MDP

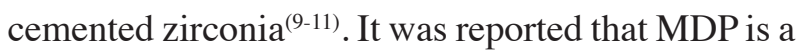
key molecule in bonding with great affinity to oxide layer on zirconia surface like $(\mathrm{ZrO} 2)$ causing an improvement in bond strength with adhesive resin substrate $^{0}$.

The adhesive potential to zirconia may be determined by other factors such as the particle size of fillers and viscosity. The active parts of MDP react with the surface of zirconia ${ }^{\circ}$.

Therefore, the bond strength may have been influenced by the resin wettability on the zirconia surface which reduces the contact angle between zirconia and adhesive resin and results in an intimate interaction between both ${ }^{(15)}$ Kim et al in $2011^{(35)}$ mentioned that the surface energy of adhesive resins and the contact angle with the bonded surface have great effect on bond strength.

Furthermore, the new rheological modifiers added to the TheraCem composition, resulted in a decrease in its viscosity compared to RelyX Unicem, where gaps were clearly seen at zirconia/ cement interface with RelyX. These indicate a higher viscosity of cement and lack of intimacy to zirconia surface ${ }^{0}$.

Nagaoka et al in $2017^{(13)}$ proposed three possible models of interaction mechanism of 10MDP with zirconia surface. It is demonstrated in figure 6 . The $1^{\text {st }}$ model on the left (figure 6a) indicates adsorbtion of the 10MD monomer onto surface by hydrogen bonding between the $\mathrm{P}=\mathrm{O}$ (oxo group) and $\mathrm{Zr}-\mathrm{OH}$ group. The second model indicates ionic bonding of 10MDP monomer with zirconia (figure 6b). The third model mentioned that in addition to ionic bonding between 10MDP and zirconia, the adsorbed 10MDP monomers have hydrogen-bonding interactions with zirconia via $\mathrm{P}=\mathrm{O}$ (oxo group) (figure $6 \mathrm{c}$ )

Many researchers mentioned that the bonding capacities of resins are related to their ability to infiltrate into substrate's surface irregularities, and that their mechanical properties are greatly affected by the percentage of fillers content. According to the manufacturers documents, the amount of silanized fillers in Rely Xunicem is $72 \mathrm{wt} \%$, while their amount in TheraCem was decreased to be only 61 $\mathrm{wt} \%$. Moreover, the presence of silanized fillers in the resin matrix decreases the viscosity ${ }^{(36,37)}$.

According to the mentioned facts, the novel cement (TheraCem) could be mechanically stronger than RelyX due to its higher substrate content, less viscosity and higher penetrating ability. Bonding performance is more related to the organic matrix than to the inorganic fillers. The latter is more responsible for mechanical properties ${ }^{(36,37)}$. 


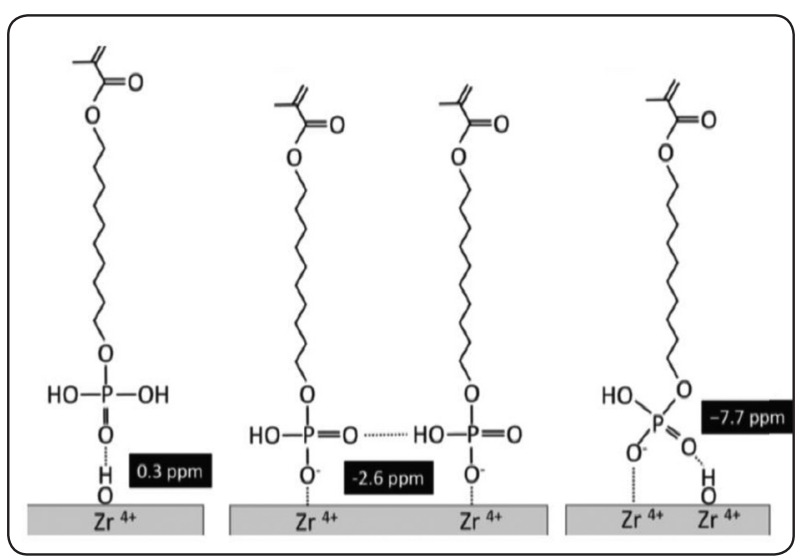

Fig. (6) A diagram explaining the proposed three different models of interactions between 10MDP and zirconia.

\section{CONCLUSIONS}

Within the limitations of the current study;

1. MDP containing resin cements can positively affect micro-shear bond strength with all tested substrates and the bond strength is not affected by thermocycling.

2. Addition of calcium and Fluoride to MDP containing new generation of cements (TheraCem) can modify the bond strength with dentin.

\section{Clinical significant:}

TheraCem self-adhesive resin cement can be considered as promising cement for many clinicians.

Further studies are required to study the unique properties of this cement and compare it with more types of self-adhesive resin cement, especially clinical trials for the new cement.

\section{REFERENCES}

1. Ranjkesh B, Ding M, Dalstra M, Nyengaard JR, Chevallier J, Isidor F, Løvschall H. Calcium phosphate precipitation in experimental gaps between fluoride-containing fast-setting calcium silicate cement and dentin. Eur J Oral Sci. 2018 Apr;126(2):118-125.

2. Rodrigues RF, Ramos CM, Francisconi PA, Borges AF. The shear bond strength of self-adhesive resin cements to dentin and enamel: an in vitro study. J Prosthet Dent. 2015 Mar;113(3):220-7
3. Vesna Miletic. Dental Composite Materials for Direct Restorations. Springe 2018:(129-155).

4. Pisani-Proença J, Erhardt MC, Amaral R, Valandro LF, Bottino MA, Del Castillo-Salmerón R. Influence of different surface conditioning protocols on microtensile bond strength of self-adhesive resin cements to dentin. J Prosthet Dent. 2011 Apr;105(4):227-35.

5. Yokota Y, Fujita KN, Uchida R, Aida E, Aoki NT, Aida M, Nishiyama N. Quantitative Evaluation of MDP-Ca Salt and DCPD after Application of an MDP-based One-step Self-etching Adhesive on Enamel and Dentin. J Adhes Dent. 2016;18(3):205-13.

6. Yaguchi T. Layering mechanism of MDP-Ca salt produced in demineralization of enamel and dentin apatite. Dent Mater. 2017 Jan;33(1):23-32.

7. Ranjkesh B, Chevallier J, Salehi H, Cuisinier F, Isidor F, Løvschall H. Apatite precipitation on a novel fast-setting calcium silicate cement containing fluoride. Acta Biomater Odontol Scand. 2016 Jan 1;2(1):68-78.

8. Shadman N1, Ebrahimi SF1, Shoul MA1, Sattari H2. In vitro evaluation of casein phosphopeptide-amorphous calcium phosphate effect on the shear bond strength of dental adhesives to enamel. Dent Res J (Isfahan). 2015 MarApr;12(2):167-72.

9. Tzanakakis EG, Tzoutzas IG, Koidis PT. Is there a potential for durable adhesion to zirconia restorations? A systematic review. J Prosthet Dent. 2016 Jan;115(1):9-19.

10. Tanaka R1, Fujishima A, Shibata Y, Manabe A, Miyazaki T. Cooperation of phosphate monomer and silica modification on zirconia. J Dent Res. 2008 Jul;87(7):666-700.

11. Inokoshi M1, De Munck J, Minakuchi S, Van Meerbeek B. Meta-analysis of bonding effectiveness to zirconia ceramics. J Dent Res. 2014 Apr;93(4):329-34.

12. Oba Y1, Koizumi H, Nakayama D, Ishii T, Akazawa $\mathrm{N}$, Matsumura H. Effect of silane and phosphate primers on the adhesive performance of a tri-n-butylborane initiated luting agent bonded to zirconia. Dent Mater J. 2014;33(2):226-32.

13. Nagaoka N1, Yoshihara K2, Feitosa VP3, Tamada Y4, Irie M5, Yoshida Y6, Van Meerbeek B7, Hayakawa S. Chemical interaction mechanism of 10-MDP with zirconia. Sci Rep. 2017 Mar 30;7:45563.

14. Murakami T, Takemoto S, Nishiyama N, Aida M. Zirconia surface modification by a novel zirconia bonding 
system and its adhesion mechanism. Dent Mater. 2017 Dec;33(12):1371-1380.

15. Grace de Souza, Diana Hennig, Anuj Aggarwal, Laura E. Ta. The use of MDP-based materials for bonding to zirconia. J Prosthet Dent 2014.

16. Keshvad A1, Hakimaneh SMR1. Microtensile Bond Strength of a Resin Cement to Silica-Based and Y-TZP Ceramics Using Different Surface Treatments. J Prosthodont. 2018 Jan;27(1):67-74.

17. Ivana Radovica/Francesca Monticellib/Cecilia Goraccic/ Zoran R. Vulicevicd/Marco Ferrar. Self-adhesive Resin Cements: A Literature Review. J Adhes Dent. 2008 Sep: 10(4): 251-258.

18. Rodrigues R, Ramos CM, Francisconi P, Borges AF.The shear bond strength of self-adhesive resin cements to dentin and enamel: an in vitro study. Prosthet Dent. 2015 Mar;113(3):220-7.

19. Shinoda Y, Nakajima M, Hosaka K, Foxton RM,Tagami J. Effect of smear layer characteristics on dentinbonding durability of HEMA-free and HEMA-containingone-step self-etch adhesives. Dent Mater 2011;30:501-10.

20. Reem Gamal, Yasser F. Gomaa, Mostafa A. Abdellatif. Microtensile bond strength and scanning electron microscopic evaluation of zirconia bonded to dentin using two selfadhesive resin cements; effect of airborne abrasion and aging.Future Dental Journal, 3(2), December 2017, 55-60.

21. Steve Armstronga, Saulo Geraldeli, Rodrigo Maiab, Luís Henrique Araújo Raposoc,Carlos José Soaresc, Junichiro Yamagawaa. Adhesion to tooth structure: A critical review of "micro" bond strength test methods. Dent Mat.2010:26:e50-62.

22. Aguiar, T.R., Vermelho, P.M., Andre, C.B., Giannini, M. Interfacial ultramorphology evaluation of resin luting cements to dentin: a correlative scanning electron microscopy and transmission electron microscopy analysis. Microsc. Res. Tech. 2013:76:1234-1239.

23. Al-Assaf, K., Chakmakchi, M., Palaghias, G., KaranikaKouma, A., Eliades, G. Interfacial characteristics of adhesive luting resins and composites with dentine. Dent. Mater.2007:23:829-839.

24. Al-Saleh, M., El-Mowafy, O. Bond strength of orthodontic brackets with new self-adhesive resin cements. Am. J. Orthod. Dentofacial Orthop. 2010:137, 528-533.

25. Braga R.R., Meira J.B., Boaro L.C., Xavier T.A. Adhesion to tooth structure: a critical review of "macro" test methods. Dent. Mater. 2010:26, e38-e49
26. Cantoro A., Goracci C., Carvalho C.A, Coniglio I., Ferrari M. Bonding potential of self-adhesive luting agents used at different temperatures to lute composite onlays. J. Dent. 2009:37:454-461.

27. Chai J., Chu F.C., Chow T.W., Liang B.M. Shear bond strength between nickel-chromium and human dentine using a dual-cure, self-adhesive universal resin luting agent. Eur. J. Prosthodont Restor Dent. 2008:16:85-88.

28. Behr M., Rosentritt M., Regnet T., Lang R., Handel G. Marginal adaptation in dentin of a self-adhesive universal resin cement compared with well tried systems. Dent. Mate.2004:20:191-197.

29. Han L., Okamoto A., Fukushima M., Okiji T. Evaluation of physical properties and surface degradation of selfadhesive resin cements. Dent. Mater. J. 2007:26:906-914.

30. Piwowarczyk A., Bender R., Ottl P., Lauer H.C. Longterm bond between dual-polymerizing cementing agents and human hard dental tissue. Dent. Mater.2007: 23:211217.

31. Nakamura T., Wakabayashi K., Kinuta S., Nishida H., Miyamae M., Yatani H. Mechanical properties of new self-adhesive resin-based cement. J. Prosthodont. Res. 2010:54:59-64.

32. Escribano N., de la Macorra J.C. Microtensile bond strength of self-adhesive luting cements to ceramic. J. Adhes. Dent. 2006:8:337-341.

33. Flury S., Lussi A., Peutzfeldt A., Zimmerli B. Push out bond strength of CAD/CAM ceramic luted to dentin with self-adhesive resin cements. Dent. Mater. 2010:26:855863.

34. Piwowarczyk A., Lauer H.C., Sorensen J.A. In vitro shear bond strength of cementing agents to fixed prosthodontics restorative materials. J. Prosthet. Dent. 2004:92,265-273.

35. Kim MJ, Kim YK, Kim KH, Kwon TY. Shearbond strengths of various luting cements to zirconia ceramic: surface chemical aspects. J Dent 2011;39:795-803.

36. Duarte Jr., S., Botta A.C., Meire M., Sadan A. Microtensile bond strengths and scanning electron microscopic evaluation of self-adhesive and self-etch resin cements to intact and etched enamel. J. Prosthet. Dent.2008:100:203-210.

37. El-Guindy, J., Selim M., El-Agroudi, M. Alternative pretreatment modalities with a self-adhesive system to promote dentin/ alloy shear bond strength. J. Prosthodont. 2010:19:205-211. 